\title{
Heyting Almost Distributive Fuzzy Lattices
}

\author{
Berhanu Assaye Alaba and Derebew Nigussie Derso
}

\begin{abstract}
In this paper, we introduce the concept of Heyting almost distributive fuzzy lattices (HADFL) using the concepts of Heyting almost distributive lattices (HADL), almost distributive fuzzy lattices, fuzzy partial order relation and fuzzy Heyting algebra. Using the properties of fuzzy Heyting algebra, we also give a necessary and sufficient condition for an HADFL to be fuzzy Heyting algebra (FHA).
\end{abstract}

Index Terms-Almost distributive fuzzy lattices, fuzzy Heyting algebra, fuzzy partial order relations, Heyting Almost distributive fuzzy Lattices.

\section{INTRODUCTION}

$\mathbf{T}$ HE concept of an almost distributive lattice (ADL) was introduced by U.M. Swamy and G.C. Rao as a common abstraction to most of the existing ring theoretic generalizations of a Boolean algebra and distributive lattices [1]. Heyting Algebra is a relatively pseudo complemented distributive lattice. It arises from non classical logic and was first investigated by Skolem T. It is named as Heyting Algebra after the Dutch Mathematician Arend Heyting [2].

Heyting almost distributive lattice was introduced by G.C. Rao, Berhanu Assaye and M.V. Ratna Mani as a generalization of a Heyting algebra in tha class of almost distributive lattices [2]. According to Zadeh [3], a fuzzy relation, which is a generalization of a function, has a natural extension to a fuzzy set and plays an important role in the theory of such sets and their applications. Similarly to an ordinary relation, a fuzzy relation in a set $X$ is a fuzzy set in the product space $X \times X$. Thus, fuzzy binary relations A are fuzzy sets of $X \times Y$ defined as a fuzzy collection of ordered pairs characterized by a membership function $\mu_{A}$ which associates with each pair (x, y) a membership degree $\mu_{A}(x, y)$ [4].

Using the concepts of bounded fuzzy lattices, fuzzy Heyting algebra(FHA) was introduced by [5]. In this paper, we introduce the concept of a Heyting almost distributive fuzzy Lattice (HADFL) using concepts of a fuzzy Heyting algebra and HADLs. We also characterize an HADFL in terms of the set of all of its principal ideals and give necessary and sufficient condition to become FHA. In the following, we give some important definitions and results that will be useful to the study of HADFLs.

\section{Preliminaries}

Definition 2.1 ([6]): A Heyting algebra is a lattice with 0 such that for any elements a, $\mathrm{b}$ there is an element $a \rightarrow b$ such that $a \wedge x \leq b$ if and only if $x \leq a \rightarrow b$.

Manuscript received October 12, 2017; accepted December 29, 2017.

B.A. Alaba is with the Department of Mathematics, College of Science, Bahir Dar University, Bahir Dar, Ethiopia. E-mail: berhanu_assaye@yahoo.com

D.N. Derso is with the Department of Mathematics, Woldia University, Woldia, Ethiopia. E-mail: nderebew@gmail.com
Definition 2.2 ([2]): An ADL $(H, \vee, \wedge, 0)$ with a maximal element is said to be a Heyting almost distributive lattice (HADL) if to each $a \in H$, the interval $[0, a]$ is a Heyting algebra.

Theorem 2.3 ([2]): The following are equivalent.

1) $\mathrm{H}$ is a Heyting algebra

2) The poset $(H, \leq)$ is directed above

3) $(H, \vee, \wedge)$ is a distributive lattice

4) $\vee$ is commutative

5) $\wedge$ is commutative

6) $\vee$ is right distributive over $\wedge$

7) The relation $\theta:=\{(a, b) \in H \times H: b \wedge a=a\}$ is antisymmetric.

Definition 2.4 ([7]): Let $\mathrm{X}$ be a set. A function A: $X \times X \rightarrow$ $[0,1]$ is called a fuzzy relation in $X$. The fuzzy relation $\mathrm{A}$ in $\mathrm{X}$ is reflexive iff $A(x, x)=1$, for all $x \in X$. The fuzzy relation $\mathrm{A}$ in $X$ is anti symmetric iff $A(x, y)>0$ and $A(y, x)>0 \Rightarrow x=y$. The fuzzy relation $\mathrm{A}$ in $\mathrm{X}$ is transitive iff $A(x, z) \geq \operatorname{Sup}_{y \in X}(\min (A(x, y), A(y, z)))$. A fuzzy relation A is fuzzy partial order relation if $\mathrm{A}$ is reflexive, symmetric and transitive. A fuzzy partial order relation A is fuzzy total order relation iff $A(x, y)>0$ or $A(y, x)>0$, for all $x, y \in \mathrm{H}$. If $\mathrm{A}$ is a fuzzy partial order relation on a set $\mathrm{X}$, then $(\mathrm{X}, \mathrm{A})$ is called a fuzzy partially ordered set or a fuzzy poset. If $\mathrm{A}$ is a fuzzy total order relation in a set $\mathrm{X}$, then $(\mathrm{X}, \mathrm{A})$ is called a fuzzy totally ordered set or a fuzzy chain.

Definition 2.5 ([5]): The fuzzy poset $(\mathrm{H}, \mathrm{A})$ is said to be directed above if $\forall a, b, c \in H, A(a, c)>0$ and $A(b, c)>0$, then $\exists x \in \mathrm{H}$ such that $A(x, c)>0$.

Definition 2.6 ([7]): Let (X,A) be a fuzzy poset and B $\subseteq \mathrm{X}$. An element $u \in \mathrm{X}$ is said to be an upper bound for a subset $\mathrm{B}$ iff $A(b, u)>0, \forall b \in \mathrm{B}$. An upper bound $u_{0}$ for a subset $\mathrm{B}$ is least upper bound of $\mathrm{B}$ iff $A\left(u_{0}, u\right)>0$ for every upper bound $u$ for B. An element $v \in \mathrm{X}$ is said to be an lower bound for a subset $\mathrm{B}$ iff $A(v, b)>0, \forall b \in \mathrm{B}$. A lower bound $v_{0}$ for a subset $\mathrm{B}$ is the greatest lower bound of $\mathrm{B}$ iff $A\left(v, v_{0}\right)>0$ for every lower bound $v$ for B. We denote the lub of the set $\{x, y\}=x \vee y$ and glb of the set $\{x, y\}=x \wedge y$

Definition 2.7: $(H, A)$ is a bounded fuzzy lattice if for any $x \in H$, we have that $A(0, x)>0$ and $A(x, 1)>0$.

Definition 2.8 ([5]): Let (H,A) be a fuzzy lattice and let $x, y, z \in H$. If $A((x \wedge y) \vee(x \wedge z), x \wedge(y \vee z))>0$ and $A(x \vee(y \wedge$ $z),(x \vee y) \wedge(x \vee z))>0$.. Then (H,A) is a distributive fuzzy lattice.

Definition 2.9: Let $(\mathrm{H}, \mathrm{A})$ is distributive fuzzy lattice.Then $(\mathrm{H}, \mathrm{A})$ is bounded if for any $x \in H$, we have that $A(0, x)>0$ and $A(x, 1)>0$.

Definition 2.10 ([6]): A bounded distributive fuzzy lattice $(\mathrm{H}, \mathrm{A})$ is said to be a Fuzzy Heyting Algebra if there exists a binary operation $\rightarrow$ such that, for any $\mathrm{x}, \mathrm{y}, \mathrm{z} \in H, A(x \wedge z, y)>$ $0 \Leftrightarrow A(z, x \rightarrow y)>0$ 
Definition 2.11 ([5]): A bounded distributive fuzzy lattice $(\mathrm{H}, \mathrm{A})$ is said to be a Fuzzy Heyting Algebra if there exists a binary operation $\rightarrow$ such that, for any $x, y, z \in H, A(x \wedge z, y)>$ $0 \Leftrightarrow A(z, x \rightarrow y)>0$

Example 2.12: Let $\mathrm{H}$ be a Heyting algebra. Then for all $a \in H,[0, a]$ is fuzzy Heyting algebra

Theorem 2.13 ([5]): Let $\mathrm{H}$ be a Heyting algebra.Then the following are equivalent:

1) $(\mathrm{H}, \mathrm{A})$ is a fuzzy Heyting algebra

2) The fuzzy poset $(\mathrm{H}, \mathrm{A})$ is directed above

3) $(\mathrm{H}, \mathrm{A})$ is a distributive fuzzy lattice

Definition $2.14([8])$ : Let $(H, \vee, \wedge, 0)$ be an algebra of type $(2,2,0)$ and $(\mathrm{H}, \mathrm{A})$ be a fuzzy poset. Then we call $(\mathrm{H}, \mathrm{A})$ is an almost distributive fuzzy Lattice (ADFL) if the following axioms are satisfied:

$(F 1) A(a, a \vee 0)=A(a \vee 0, a)=1$

$(F 2) A(0,0 \wedge a)=A(0 \wedge a, 0)=1$

$(F 3) A((a \vee b) \wedge c,(a \wedge c) \vee(b \wedge c))=A((a \wedge c) \vee(b \wedge c),(a \vee$

b) $\wedge c)=1$

$(F 4) A(a \wedge(b \vee c),(a \wedge b) \vee(a \wedge c))=A((a \wedge b) \vee(a \wedge c), a \wedge$ $(b \vee c))=1$

$(F 5) A(a \vee(b \wedge c),(a \vee b) \wedge(a \vee c))=A((a \vee b) \wedge(a \vee c), a \vee$ $(b \wedge c))=1$

$(F 6) A((a \vee b) \wedge b, b)=A(b,(a \vee b) \wedge b)=1$ for all $a, b, c \in H$.

\section{Heyting Almost Distributive Fuzzy Lattice}

In this section, we introduce the concept of a Heyting almost distributive fuzzy lattice and study some important properties.

Definition 3.1: An ADFL $L=(H, A)$ with maximal element $m$ is said to be Heyting almost distributive fuzzy lattices if to each $a \in H$, the interval $[0, a]$ is fuzzy Heyting algebra.

Example 3.2: Every FHA is an HADFL since every interval in FHA is itself FHA.

Theorem 3.3: Let $(\mathrm{H}, \mathrm{A})$ be an HADFL with a maximal element $\mathrm{m}$. Then the following are equivalent.

(1) $\mathrm{H}$ is an HADFL

(2) $[0, m]$ is a Fuzzy Heyting algebra

(3) There exists a binary operation $\rightarrow$ on $\mathrm{H}$ such that the following conditions hold: for all $\mathrm{x}, \mathrm{y}, \mathrm{z} \mathrm{H}$,

(i) $A(m, x \rightarrow x)>0$

(ii) $A(y,(x \rightarrow y) \wedge y)>0$

(iii) $A(x \wedge y \wedge m, x \wedge(x \rightarrow y))>0$

(iv) $A((x \rightarrow(y \wedge z),(x \rightarrow y) \wedge(x \rightarrow z))=A((x \rightarrow y) \wedge(x \rightarrow$ $z), x \rightarrow(y \wedge z))=m$

(v) $A((x \vee y) \rightarrow z,(x \rightarrow z) \wedge(y \rightarrow z))=A((x \rightarrow z) \wedge(y \rightarrow$ $z)),(x \vee y) \rightarrow z)=m$

Proof: Let $\mathrm{H}$ be an HADFL with 0 and a maximal element $\mathrm{m}$. Let $\rightarrow$ be a binary operation on $\mathrm{H}$. (1) $\Rightarrow(2)$ is trivial .

$(2) \Rightarrow(3)$ : Assume that $[0, \mathrm{~m}]$ is a fuzzy Heyting algebra in which the binary operation $(\rightarrow)$ is denoted by $\rightarrow_{m}$. For $x, y \in$ $\mathrm{H}$, define $x \rightarrow y=x \wedge m \rightarrow_{m} x \wedge m$. Let $x, y, z \in \mathrm{H}$.

Now (i) $A(m, x \rightarrow x)=A\left(m, x \wedge m \rightarrow_{m} x \wedge m\right)=A(m, m)>0$

(ii) $A(y,(x \rightarrow y) \wedge y)=A\left(y,\left(x \wedge m \rightarrow_{m} y \wedge m\right) \wedge y\right)=A(y,(x \wedge$ $\left.\left.m \rightarrow_{m} y \wedge m\right) \wedge y \wedge m\right)=A(y, y \wedge m)>0$. Routinely we can show others. (3) $\Rightarrow(1)$ Assume the conditions hold on $H$,then for $a \in H$.We know that $[0, a]$ is a distributive fuzzy lattice. Define a binary operation $\rightarrow_{a}$ on $[0, a]$ by $x \rightarrow_{a} y=(x \rightarrow y) \wedge a, \forall x, y \in$ $[0, a]$.

Let $x, y, z \in[0, a]$ and $A(x \wedge z, y)>0$.Then $A(x \rightarrow(x \wedge z), x \rightarrow$ $y)>0$.

$\Rightarrow A(m \wedge(x \rightarrow z), x \rightarrow y)>0$

$\Rightarrow A(x \rightarrow z, x \rightarrow y)>0$

$\Rightarrow A((x \rightarrow z) \wedge a,(x \rightarrow y) \wedge a)>0$ [by property ]

$\Rightarrow A\left((x \rightarrow z) \wedge a, x \rightarrow_{a} y\right)>0$

$\Rightarrow A\left(z \wedge(x \rightarrow z) \wedge a, z \wedge\left(x \rightarrow_{a} y\right)\right)>0$

$\Rightarrow A((x \rightarrow z) \wedge z \wedge a, z \wedge(x \rightarrow a y))>0$

$\Rightarrow A\left(z \wedge(x \rightarrow z) \wedge a, x \rightarrow_{a} y\right)>0\left[\right.$ since $z \wedge\left(x \rightarrow_{a} y\right) \leq x \rightarrow_{a} y$ ]

$\Rightarrow A\left(z \wedge a, x \rightarrow_{a} y\right)>0$

Thus, $A\left(z, x \rightarrow_{a} y\right)>0$ [Since $z \leq a$ ]

Conversely, assume that $z \in[0, a]$ and $A\left(z, x \rightarrow_{a} y\right)>0$.

$\Rightarrow A(x \wedge z, x \wedge(x \rightarrow y) \wedge a)>0$.

$\Rightarrow A(x \wedge z, x \wedge y \wedge a)>0$.

$\Rightarrow A(x \wedge z, x \wedge y)>0$.

$\Rightarrow A(x \wedge z, y)>0$. [Since $A(x \wedge y, y)>0]$

Thus, $[0, \mathrm{a}]$ is a fuzzy Heyting algebra.

Therefore, $\mathrm{H}$ is an HADFL.

Through out this section the symbol $\mathrm{H}$ stands for an HADFL $(\mathrm{H}, \mathrm{A})$ unless otherwise specified. In the following lemma, we give some important properties of HADFL.

Lemma 3.4: Let $x, y, a \in H$ and $A(x, y)>0$. Then the following hold:

1) $A(a \rightarrow x, a \rightarrow y)>0$

2) $A(y \rightarrow a, x \rightarrow a)>0$

3) $A(m,(a \wedge b) \rightarrow b)>0$

Proof:

(1) Let $\mathrm{x}, \mathrm{y}, \mathrm{a} \in H$ and $A(x, y)>0$. Since $\mathrm{H}$ is an HADFL, $A((a \rightarrow x) \wedge(a \rightarrow y), a \rightarrow(x \wedge y))=1$

$A((a \rightarrow x) \wedge(a \rightarrow y), a \rightarrow x)=1$. $[\operatorname{Since} A(x, y)>0 \Leftrightarrow x \wedge y=x]$.

This implies $a \rightarrow x=(a \rightarrow x) \wedge(a \rightarrow y)$

$\Rightarrow A(a \rightarrow x, a \rightarrow y)>0$

(2) $A((y \rightarrow a) \wedge(x \rightarrow a),(y \vee x) \rightarrow) a=1$

$A((y \rightarrow a) \wedge(x \rightarrow a), y \rightarrow a)=1$

$\Rightarrow A(y \rightarrow a, x \rightarrow a)>0$

(3) $A(a \wedge b, b)>0$

$\Rightarrow A((a \wedge b) \rightarrow(a \wedge b),(a \wedge b) \rightarrow b)>0$

$\Rightarrow A(m,(a \wedge b) \rightarrow b)>0$.

\section{PROPERTIES OF HADFL}

Theorem 4.1: For any $x, y \in H$,we have the following.

(1) $A(y, x \rightarrow 0)>0 \Rightarrow x \wedge y=0$

(2) $A(y \rightarrow 0, x \rightarrow 0)>0 \Leftrightarrow x \wedge(y \rightarrow 0)=0$

(3) $A(m \rightarrow x, x)>0$

(4) $A(x \rightarrow m, m)>0$

Proof: (1) $A(y, x \rightarrow 0)>0$

$\Rightarrow A(x \wedge y, x \wedge(x \rightarrow 0))>0$

$\Rightarrow A(x \wedge y, x \wedge 0)>0$. This implies $x \wedge y \leq 0$. But $0 \leq x \wedge y$

Thus, $x \wedge y=0$

$(2)(\Rightarrow) A(x \wedge(y \rightarrow 0), x \wedge(x \rightarrow 0))>0$

$\Rightarrow A(x \wedge(y \rightarrow 0), x \wedge 0 \wedge m)>0$

$\Rightarrow x \wedge(y \rightarrow 0)=0$. Conversely, assume $x \wedge(y \rightarrow 0)=0$. Then

$A(y \rightarrow 0, x \rightarrow 0)$

$=A(y \rightarrow 0, x \rightarrow(x \wedge(y \rightarrow 0)))$ 


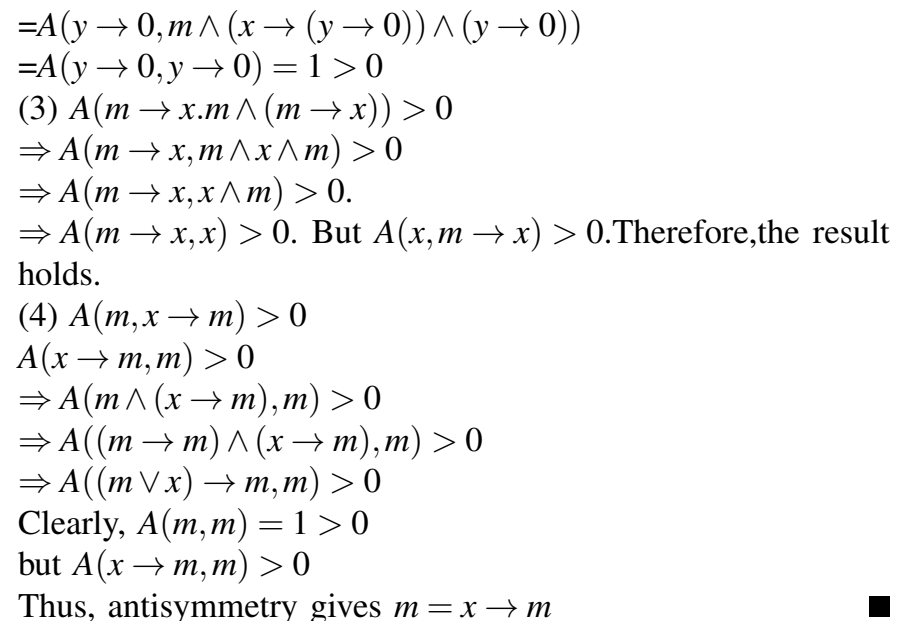

Thus, antisymmetry gives $m=x \rightarrow m$

Lemma 4.2: $A(x \wedge y, x)=1 \Leftrightarrow A(x, y)>0$.

Theorem 4.3: Let $\mathrm{m}$ be the maximal element in $\mathrm{H}$. Then for any a,b,c $\in \mathrm{H}$,the following holds.

1) $A(b \wedge m,(a \rightarrow b) \wedge m))>0$

2) $A(a \wedge m, b \wedge m)>0 \Leftrightarrow(a \rightarrow b) \wedge m=m$

3) $A(a \wedge b \wedge m, a \wedge c \wedge m)=1 \Leftrightarrow A((a \rightarrow b) \wedge m,(a \rightarrow c) \wedge$ $m)=1$

4) $A(a \wedge c \wedge m, b \wedge m)>0 \Leftrightarrow A(c \wedge m,(a \rightarrow b) \wedge m)>0$

5) $A(a \wedge m,[(a \rightarrow b) \rightarrow b] \wedge m)>0$

6) $A(a \wedge m,(b \rightarrow c) \wedge m)>0 \Leftrightarrow A(b \wedge m,(a \rightarrow c) \wedge m)>0$

Proof: (1) Since $A(b, a \rightarrow b)>0 A(m, m)>0$. Then, $A(b \wedge$ $m,(a \rightarrow b) \wedge m)>0$.

(2) $A(a \wedge m, b \wedge m)>0$

$\Rightarrow A(a \rightarrow(a \wedge m), a \rightarrow(b \wedge m))>0$

$\Rightarrow A((a \rightarrow a) \wedge(a \rightarrow m),(a \rightarrow b) \wedge(a \rightarrow m))>0$.

$\Rightarrow A(m \wedge(a \rightarrow m), a \rightarrow(b \wedge m))>0$

$\Rightarrow A(m, a \rightarrow(b \wedge m))>0$ [Since $a \rightarrow m=m$, Theorem 3.1]

But $A((a \rightarrow b) \wedge m, m)>0$. Anti symmetry property gives $(a \rightarrow$ b) $\wedge m=m$ Conversely, $A((a \rightarrow b) \wedge m, m)=1, A(a, a)>0$.

$\Rightarrow A(a \wedge(a \rightarrow b) \wedge m), a \wedge m)=1$

$\Rightarrow A(a \wedge b \wedge m, a \wedge m)=1$.

$\Rightarrow A(a \wedge m \wedge b \wedge m, a \wedge m)=1$.

$\Rightarrow A(a \wedge m, b \wedge m)>0$. [ lemma 1.1.2]

(3)Assume that $A(a \wedge b \wedge m, a \wedge c \wedge m)=1>0$.

By (2) $A(a \rightarrow(a \wedge b \wedge m), a \rightarrow(a \wedge c \wedge m))>0$. Hence $A((a \rightarrow$ b) $\wedge m,(a \rightarrow c) \wedge m$. $)>0$

Conversely, assume that $A((a \rightarrow b) \wedge m,(a \rightarrow c) \wedge m)>0$. $A(a \wedge(a \rightarrow b) \wedge m, a \wedge(a \rightarrow c) \wedge m)>0$. Hence $A(a \wedge b \wedge m, a \wedge$ $c \wedge m)>0$.

(4). Assume $A(a \wedge c \wedge m, b \wedge m)>0$.

$\Rightarrow A(a \rightarrow(a \wedge c \wedge m), a \rightarrow(b \wedge m))>0$.

$\Rightarrow \mathrm{A}(a \rightarrow(c \wedge m),(a \rightarrow b) \wedge(a \rightarrow m))>0$.

$\Rightarrow A((a \rightarrow c) \wedge m,(a \rightarrow b) \wedge m)>0$ [Theorem 3.1]

$\Rightarrow A(c \wedge m,(a \rightarrow b) \wedge m)>0$ [By 1 above]

This gives $A(a \wedge(c \wedge m), a \wedge(a \rightarrow b) \wedge m)>0$, and hence $A(a \wedge c \wedge m, a \wedge b \wedge m)>0$. Thus, $A(a \wedge c \wedge m, b \wedge m)>0$.

(5) Now $A(a \wedge(a \rightarrow b) \wedge m, a \wedge b \wedge m)>0$

$\Rightarrow A(a \wedge(a \rightarrow b) \wedge m, b \wedge m)>0$

$\Rightarrow A((a \rightarrow b) \wedge a \wedge m, b \wedge m)>0$

$\Leftrightarrow A(a \wedge m,[(a \rightarrow b) \rightarrow b] \wedge m)>0[$ By definition ]

(6) Assume that $A(a \wedge m,(b \rightarrow c) \wedge m)>0$.

$\Rightarrow A(b \wedge a \wedge m, b \wedge(b \rightarrow c) \wedge m)>0$

$\Rightarrow A(b \wedge a \wedge m, b \wedge c \wedge m)>0$ and $A(b \wedge c \wedge m, c \wedge m)>0$
$\Rightarrow A(b \wedge a \wedge m, c \wedge m)>0$

Therefore, $A(b \wedge m,(a \rightarrow c) \wedge m)>0$. Conversely, assume $A(b \wedge m,(a \rightarrow c) \wedge m)>0$

$\Rightarrow A(a \wedge b \wedge m, a \wedge(a \rightarrow c) \wedge m)>0$

$\Rightarrow A(a \wedge b \wedge m, a \wedge c \wedge m)>0$

$\Rightarrow A(a \wedge b \wedge m, c \wedge m)>0$

$\Rightarrow A(b \wedge a \wedge m, c \wedge m)>0$

Thus, $A(a \wedge m,(b \rightarrow c) \wedge m)>0$.

\section{Characterization OF HADFL}

Definition 5.1: Let $(H, A)$ be an HADFL $a \in H$, then the principal ideal generated by a is denoted by $(a]_{A}$ and is equal to $\{x \in H: A(x, a \wedge x)>0\}$. We denote the set of all principal ideals of $\mathrm{H}$ by $\mathrm{PI}(\mathrm{H})$.

Lemma 5.2: If $(a] \subseteq(b]$, then $(a]_{A} \subseteq(b]_{A}$, for all $a, b \in H$.

Lemma 5.3: $a \in(b] \Leftrightarrow A(a, b \wedge a)>0$.

Lemma 5.4: Let $a, b \in H$ and (H,A) be an HADFL be an HADFL, then the following are equivalent.

1) $(a]_{A} \subseteq(b]_{A}$

2) $A(a, b \wedge a)>0$

3) $A(a \wedge x, b \wedge x)>0, \forall x \in H$

Theorem 5.5: Let $(\mathrm{H}, \mathrm{A})$ be an ADFL with 0 and a maximal element $\mathrm{m}$,then $(\mathrm{H}, \mathrm{A})$ is an HADFL iff $(\mathrm{PI}(\mathrm{H}), \mathrm{A})$ is a FHA .

Proof: Suppose $(\mathrm{H}, \mathrm{A})$ be an HADFL. Then $(\mathrm{PI}(\mathrm{H}), \mathrm{A})$ is a distributive fuzzy lattice. For any $x, y \in H$, define $(x] \rightarrow$ $(y]=(x \rightarrow y]$. If $(a]=(b]$, and $(c]=(d]$. Then $A(b, a \wedge b)>$ $0, A(a, b \wedge a)>0, A(d, c \wedge d)>0, A(c, d \wedge c)>0$.

Consider $A(b \rightarrow d,(b \rightarrow c) \wedge(b \rightarrow d))$

$=A(b \rightarrow d,(b \vee a) \rightarrow c) \wedge(b \rightarrow d))$

$=A(b \rightarrow d,(b \rightarrow c) \wedge(a \rightarrow c) \wedge(b \rightarrow d))$

$>0$. Again $A((b \rightarrow c) \wedge(a \rightarrow c) \wedge(b \rightarrow d),(a \rightarrow c) \wedge(b \rightarrow$ d)) $>0$

$\Rightarrow A(b \rightarrow d,(a \rightarrow c) \wedge(b \rightarrow d))>0$ and $A((a \rightarrow c) \wedge(b \rightarrow$ d), $b \rightarrow d))>0$.

$\Rightarrow A(b \rightarrow d,(a \rightarrow c) \wedge(b \rightarrow d))>0$. By lemma 5.3 we have $(a \rightarrow c] \subseteq(b \rightarrow d]$, by symmetry, $(b \rightarrow d] \subseteq(a \rightarrow c]$. Thus, $(a \rightarrow c]=(b \rightarrow d]$ Therefore, " $\Rightarrow$ " is well defined on $\mathrm{PI}(\mathrm{H})$. By theorem on FHA, a bounded distributive fuzzy lattice is a FHA. Conversely, assume $\mathrm{PI}(\mathrm{H})$ is a FHA. For $a, b \in H$, define $a \rightarrow b=c \wedge m$, where $(a] \rightarrow(b]=(c]$ for some $c \in H$. Let $(s]=(t]$ for some $s, t \in H$. Then $A(s \wedge t, t)>0, A(t, s \wedge t)>$ $0, A(s, t \wedge s)>0$ and $A(t \wedge s, s)>0$

$\Rightarrow A(s \wedge m, t \wedge s \wedge m)>0$

$\Rightarrow A(s \wedge m, s \wedge t \wedge m)>0$.

But $A(t \wedge s \wedge m, s \wedge m)>0$

$\Rightarrow t \wedge s \wedge m=s \wedge m$ [since antisymmetry]

$\Rightarrow s \wedge t \wedge m=s \wedge m$

$\Rightarrow t \wedge m=s \wedge m$

Thus the binary operation " $\rightarrow$ " is well defined. Let $a, b, c \in H$. We prove that $(H, A)$ is HADFL.

(1) since $(a] \rightarrow(a]=(m]$. Then ew get $a \rightarrow a=m \wedge m$

$\Rightarrow a \rightarrow a=m$

$\Rightarrow A(m, a \rightarrow a)>0$

(2) Let $(a] \rightarrow(b]=(c]$. Then $(a \rightarrow b) \wedge b=c \wedge m \wedge b=c \wedge b=$ $b$.Thus, $A((a \rightarrow b) \wedge b, b)>0$.

(3) since $(a] \rightarrow(b]=(c]$. Then $(a] \wedge(c]=(a] \wedge((a] \rightarrow(b])$ $\Rightarrow(a \wedge c]=(a \wedge b]$. Now $(a \wedge(a \rightarrow b))=a \wedge c \wedge m=a \wedge b \wedge m$ 
$\Rightarrow A(a \wedge(a \rightarrow b), a \wedge b \wedge m)>0$. and $A(a \wedge b \wedge m, a \wedge(a \rightarrow b)>$ 0

(4) Let $(a] \rightarrow(c]=(t]$ and $(b] \rightarrow(c]=(s]$. Then $a \rightarrow c=t \wedge m$ and $b \rightarrow c=s \wedge m$.Consider, $(a \vee b] \rightarrow(c]=((a] \vee(b]) \rightarrow(c]=$ $((a] \rightarrow(c]) \wedge((b] \rightarrow(c])=(t] \wedge(s]=(t \wedge s]$.

$\Rightarrow(a \vee b) \rightarrow c=t \wedge s \wedge m=t \wedge m \wedge s \wedge m=(a \rightarrow c) \wedge(b \rightarrow c)$ $A((a \vee b) \rightarrow c,(a \rightarrow c) \wedge(b \rightarrow c))>0$ and $A((a \rightarrow c) \wedge(b \rightarrow$ $c),(a \vee b) \rightarrow c)>0$

(5) similar to (4) we can prove that $A(a \rightarrow(b \wedge c),(a \rightarrow c) \wedge$ $(a \rightarrow c))>0$ and $A((a \rightarrow c) \wedge(a \rightarrow c), a \rightarrow(b \wedge c))>0$.

Thus, (H,A) is an HADFL.

Now, we give another characterization for an HADFL to become a FHA.

Theorem 5.6: Let $(\mathrm{H}, \mathrm{A})$ be an HADFL.Then $(\mathrm{H}, \mathrm{A})$ is a FHA iff for any $a \in H, \theta_{a}=\{(x, y) \in H \times H: A(a,(x \rightarrow y) \wedge(y \rightarrow$ $x)>0\}$ is a congruence relation on $\mathrm{H}$.

Proof: Assume that (H,A) is a FHA and $a \in H$.

1) $\theta_{a}$ is reflexive. $\operatorname{since} A(a,(a \rightarrow a) \wedge(a \rightarrow a))=A(a, m)>$ 0 [ as m maximal in $\mathrm{H}$ ].

Thus, $(a, a) \in \theta_{a}$

2) $\theta_{a}$ is symmetric. Let $(x, y) \in \theta_{a}$.

Then $A(a,(x \rightarrow y) \wedge(y \rightarrow x))>0$ $\Rightarrow A(a,(y \rightarrow x) \wedge(x \rightarrow y))>0$ [ By hypothesis]

3) $\theta_{a}$ is transitive. Let $x, y, z \in H$ such that $(x, y) \in \theta_{a},(y, z) \in$ $\theta_{a}$, then $A(a,(x \rightarrow y) \wedge(y \rightarrow x))>0$ and $A(a,(y \rightarrow z) \wedge$ $(z \rightarrow y))>0$

$\Rightarrow A(a,(x \rightarrow y) \wedge(y \rightarrow x) \wedge(y \rightarrow z) \wedge(z \rightarrow y))>0 . *$

$\theta_{a}$ is transitive Since , $x \wedge(x \rightarrow y) \wedge(y \rightarrow z)=x \wedge y \wedge z \leq$ $z$, we have $A(x \wedge(x \rightarrow y) \wedge(y \rightarrow z), z)>0$.

$\Rightarrow A((x \rightarrow y) \wedge(y \rightarrow z), x \rightarrow z)>0$. **[From definition of FHA]

Similarly, Again $z \wedge(z \rightarrow y) \wedge(y \rightarrow x)=z \wedge y \wedge x \leq x$, we have $A(z \wedge(z \rightarrow y) \wedge(y \rightarrow x), x)>0$.

$\Rightarrow A((z \rightarrow y) \wedge(y \rightarrow x), z \rightarrow x)>0 * * *$

From ** and ***, we have $A((x \rightarrow y) \wedge(y \rightarrow z) \wedge(z \rightarrow$ $y) \wedge(y \rightarrow x),(x \rightarrow z) \wedge(z \rightarrow x))>0$. **** Thus, from * and $* * * *$ we have $A(a,(x \rightarrow z) \wedge(z \rightarrow x))>0$. Therefore, $(x, z) \in \theta_{a}$ From (1), (2) and (3) $\theta_{a}$ is an equivalence relation on $\mathrm{H}$.

4) $\theta_{a}$ is a congruence relation.

Since $A(x \wedge d, x)>0$ and $A(y \wedge d, y)>0$, we have $A(x \rightarrow y,(x \wedge d) \rightarrow y)>0$ and $A(y \rightarrow x,(y \wedge d) \rightarrow x)>0$. $\Rightarrow A((x \rightarrow y) \wedge(y \rightarrow x),(x \wedge d) \rightarrow y) \wedge(y \wedge d) \rightarrow x))>$ 0 .But $A(a,(x \rightarrow y) \wedge(y \rightarrow x))>0$. This gives $A(a,(x \wedge$ $d) \rightarrow y) \wedge(y \wedge d) \rightarrow x))>0$. Thus, $(x \wedge d, y \wedge d) \in \theta_{a}$. Вy similar argument, we can show that $(x \vee d, y \vee d) \in \theta_{a}$. Now, $A(x \wedge(x \rightarrow y) \wedge(y \rightarrow d), d)>0$. This implies $A((x \rightarrow y) \wedge(y \rightarrow d),(x \rightarrow d))>0$.

$\Rightarrow A((x \rightarrow y),(y \rightarrow d) \rightarrow(x \rightarrow d))>0$. Also by symmetry, $A((y \rightarrow x),(x \rightarrow d) \rightarrow(y \rightarrow d))>0$

$\Rightarrow A((x \rightarrow y) \wedge(y \rightarrow x),((y \rightarrow d) \rightarrow(x \rightarrow d)) \wedge((x \rightarrow$ $d) \rightarrow(y \rightarrow d)))>0 \ldots * * 1$

Using $A(a,(x \rightarrow y) \wedge(y \rightarrow x))>0$ and $* 1$ we have $A(a,(y \rightarrow d) \rightarrow(x \rightarrow d)) \wedge((x \rightarrow d) \rightarrow(y \rightarrow d)))>$ 0.Thus, $(x \rightarrow d, y \rightarrow d) \in \theta_{a}$. Similarly one can show that $(d \rightarrow x, d \rightarrow y) \in \theta_{a}$. Hence the result.

Conversely, assume that $\mathrm{H}$ is an HADFL in which $\theta_{a}$ is a congruence relation on $\mathrm{H}$ for all $a \in H$. Now for any $a \in$
$H,(a, a)$ is $\theta_{a}$, we get $A(a, a \rightarrow a)>0$. This implies $A(a, m)>$ 0

$(H, A)$ is a distributive fuzzy lattice and hence it is a FHA (by Theorem 2.7). Finally summing up all the characterization of an HADFL $(\mathrm{H}, \mathrm{A})$ to become a FHA.

We state the following theorem.

Theorem 5.7: In an HADFL (H,A),the follwing are equivalent

1) $(\mathrm{H}, \mathrm{A})$ is FHA.

2) $(\mathrm{H}, \mathrm{A})$ is Distributive fuzzy lattice.

3) The fuzzy poset $(\mathrm{H}, \mathrm{A})$ is directed above

4) For $a, b, c \in H, A(a \wedge c, b)>0 \Leftrightarrow A(c, a \rightarrow b)>0$

5) $A(b, a \rightarrow b)>0$,for all $a, b \in H$

6) $\theta_{a}=\{(x, y) \in H \times H: A(a,(x \rightarrow y) \wedge(y \rightarrow x))>0\}$ is a congruence relation on $H, \forall a \in H$.

\section{REFERENCES}

[1] U. Swamy and G. Rao, "Almost distributive lattices," Journal of the Australian Mathematical Society, vol. 31, no. 1, pp. 77-91, 1981.

[2] G. Rao, B. Assaye, and M. Mani, "Heyting almost distributive lattices," Inter. J. Computational Cognition, vol. 8, no. 3, pp. 85-89, 2010.

[3] L. Zadeh, "Information and control," Fuzzy sets, vol. 8, no. 3, pp. 338353, 1965.

[4] I. Mezzomo, "On fuzzy ideals and fuzzy filters of fuzzy lattices," Ph.D. dissertation, Universidade Federal do Rio Grande do Norte, Dec. 2013.

[5] B. Alaba and D. Derso, Fuzzy Heyting Algebra. Springer, Cham, 2018, pp. 25-33.

[6] R. Balbes and A. Horn, "Injective and projective heyting algebras," Transactions of the American Mathematical Society, vol. 148, no. 2, pp. 549-559, 1970

[7] I. Chon, "Fuzzy partial order relations and fuzzy lattices," Korean J. Math, vol. 17 , no. 4, pp. 361-374, 2009.

[8] B. Assaye, Y. Gedamu, and B. Tarekegn, "Almost distributive fuzzy lattices," International Journal of Mathematics and Its Applications, vol. 5, no. 1-C, pp. 307-316, 2017. 